\title{
P155
}

\section{A Low-frequency Apparatus for Characterizing the Mechanical Properties of Rocks}

V. Mikhaltsevitch* (Curtin University), M. Lebedev (Curtin University) \& B. Gurevich (Curtin University)

\section{SUMMARY}

We present a new version of the low frequency laboratory apparatus operating at seismic frequencies and at either confining or uniaxial pressures from 0 to $70 \mathrm{Mpa}$. The apparatus utilizes a stress-strain technique and measures the complex Young's modulus, extensional attenuation and Poisson's ratio of the rock sample at the range of strain amplitudes between 10-6 and 10-8. 


\section{Dienna '11}

\section{Introduction}

One of the crucial problems of rock physics modelling arises from the fact that ultrasonic devices, which are the most extensively used laboratory apparatus for determination of the rock elastic parameters, operate at frequencies from several hundred $\mathrm{kHz}$ to the $\mathrm{MHz}$ region (Ellis and Singer, 2008). However, the dominant frequencies of the seismic waves with sufficient energy for recording in exploration seismology lie in a range from $2 \mathrm{~Hz}$ to $120 \mathrm{~Hz}$ (Sheriff and Geldart, 1995).

The laboratory devices based on acoustic resonance techniques utilize the relationship between the elastic properties and the resonance frequencies of the examination object. The resonance frequencies are determined by the boundary conditions and therefore are dependent on the dimensional sizes and shape of the object what crucially limits the range of acoustic frequencies the elastic properties could be extracted at and makes a range of a few hertz unachievable (Weidner, 1987).

Consequently, the results of the ultrasonic and "resonant bar" methods cannot be directly extrapolated for seismic data interpretation.

The low frequency devices proposed in some papers (Spencer, 1981; Paffenholz and Burkhardt, 1989; Batzle at al, 2006) utilize a stress-strain technique and measure the complex Young's moduli of the solid samples at seismic frequencies. However, only the device proposed by Batzle at al (2006) is able to operate at confining pressures, and none of the devices operate at high uniaxial pressures applied to the sample.

In this paper we present the preliminary data obtained with a new low-frequency laboratory apparatus operating at seismic frequencies. This apparatus measures the complex Young's moduli of the rocks at confining or uniaxial pressures from 0 to $70 \mathrm{MPa}$.

\section{Apparatus}

An essential disadvantage of the apparatus described in paper by Batzle et al (2006) arises from the fact of using the electromechanical shaker as a source of axial dynamic displacements in a sample. The Young's modulus is measured as the ratio of the axial stress, applied to the sample by the shaker, to the resulting strain in the sample directly monitored by sensitive strain gauges. To apply a confining pressure to the sample the device is immersed into a pressure vessel. Because the electromagnetic shaker is unable to work under high load, uniaxial pressure cannot essentially exceed the pressure created by the gas in the pressure vessel and applied to the whole mechanical assembly of the apparatus. The last factor makes the experiments with separated uniaxial and confining pressures unfeasible.

In this paper we present a new version of the low-frequency apparatus which measures the complex Young's moduli of the rock samples at frequencies from $0 \mathrm{~Hz}$ to $20 \mathrm{kHz}$ and at uniaxial or confining pressures from 0 to $70 \mathrm{MPa}$. The mechanical assembly of the apparatus is presented in Figure 1 . The assembly comprises two massive platforms 1 and 2 placed on the top and in the bottom of the apparatus, and a set of units between the platforms which includes a hydraulic pressure machine 2, a tri-axial core holder 6, a piezoelectric actuator 3, aluminum calibration standard 4, and two plugs 5 and 9 having passages for a fluid injection.

The replacement of the electromechanical shaker by the piezoelectric actuator enables us to apply a high uniaxial pressure to the sample. We used APC International piezoelectric actuator (type Pst 1000/35/40) with a maximum load of $70 \mathrm{kH}$ and a frequency range from 0 to $25 \mathrm{kHz}$.

The main purpose of using the platforms 1 and 2 is a reduction of the vibrations of the apparatus caused by acoustic resonances in the mechanical assembly. The effect of vibration reduction is enhanced by the hydraulic pressure machine 2 due to damping properties of the fluid in the body of the pressure machine.

A specimen to be tested 7 is placed inside a sleeve 8 , which is mounted within the central passage 13 of the tri-axial core holder 6 . The sleeve 8 is made of elastomer, however, other resilient material may be used. Two plugs 5 and 9 ensure the flow of fluids through the specimen 7 and provide the means for pore pressure control. The central passage 13 of the core holder 6 and pressure machine 2 are connected via fluid lines with two independent pressure sources providing radial and axial forces applied to the specimen. Therefore one can vary the axial and radial forces independently of each 
other what is very important for studying the physical characteristics of the specimen under various conditions.

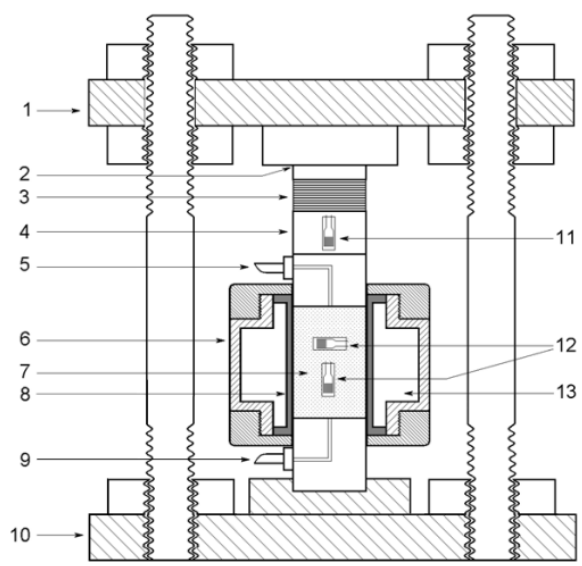

Figure 1: The mechanical assembly of the low-frequency apparatus.

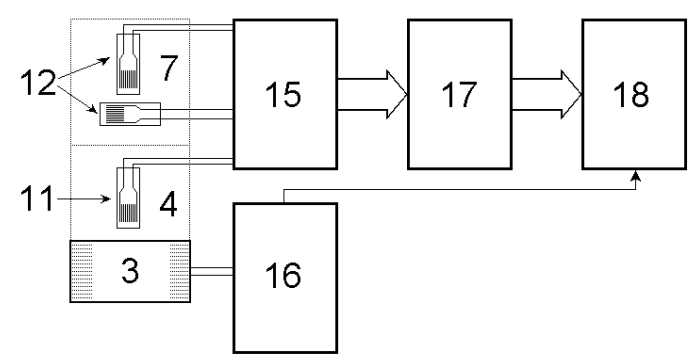

Figure 2: The electronic schematics of the low-frequency apparatus.

The schematic diagram of the electrical part of the apparatus is shown in Figure 2. The multilayer piezoelectric actuator 3 transforms the periodic voltage, applied by an oscillator 16, into mechanical stress, which causes displacements in the aluminum standard 4 and specimen 7 . The displacements modulate the conductivity of the strain gauges 11 and 12 coupled with the aluminum standard 4 and specimen 7 correspondingly. A set of electrical bridges 15 transforms the modulated conductivity into electrical signals, which, after digitizing by an analogue-digital converter 17 , are received by an acquisition computer 18, where the signals are averaged and processed. The processing in the acquisition computer 18 is synchronized with the oscillator 16 by a special triggering signal.

\section{Method and Operation}

Now we describe the process of measuring in more detail. The signals corresponding to the axial and radial components of the strain in the specimen 7 are detected by the strain gauges 12 , one of which is aligned with axial direction and the second is orthogonal to the first one. These signals are used to calculate the Young's modulus and Poisson's ratio.

If we assume that stress is applied along z-axis, then from Hooke's law (e.g., Mavko et al, 2009)

$$
\varepsilon_{i j}=\frac{1}{E}(1+v) \sigma_{i j}-v \delta_{i j} \sigma_{\alpha \alpha},
$$

where $\varepsilon_{i j}$ are the elements of the specimen strain tensor; $\sigma_{i j}$ are the elements of the stress tensor; $\sigma_{\alpha \alpha}=\sum_{i=1}^{3} \sigma_{i i}$; we can find the Poisson's ratio $v$

$$
v=\left(c-\frac{\varepsilon_{x x}}{\varepsilon_{z z}}\right) /\left(1+c-2 c \cdot \frac{\varepsilon_{x x}}{\varepsilon_{z z}}\right) \text {, where } c=\frac{\sigma_{x x}}{\sigma_{z z}} .
$$

The Young's modulus of the specimen $E$ can be found from Eq. (1): 


$$
E=\sigma_{z z} \frac{(1+v)(1-2 v)}{2 v \varepsilon_{x x}+(1-v) \varepsilon_{z z}},
$$

The stress $\sigma_{z z}$ can be expressed through the parameters of the aluminium standard as $E_{a l} \varepsilon_{z z}^{a l}$, where $E_{a l}$ is the known Young's modulus and $\varepsilon_{z z}^{a l}$ is a measured amplitude of axial strain. So, the Young's modulus of the specimen is

$$
E=E_{a l} \frac{\varepsilon_{z z}^{a l}(1+v)(1-2 v)}{2 v \varepsilon_{x x}+(1-v) \varepsilon_{z z}} .
$$

It can be shown that the coefficient $c$, which is required for the determination of the Poisson's ratio $v$ in Eq. (2), is equal to

$$
k_{l} \frac{r L_{s}}{\left(R^{2}-r^{2}\right) L \cdot E_{\mathrm{al}}} \frac{\varepsilon_{x x}}{\varepsilon_{z z}^{a l}},
$$

where $k_{l}$ is the bulk modulus of the oil; $L_{s}$ and $r$ are the length and radius of the specimen; $\mathrm{R}$ is the internal radius of the core holder.

Using the Young's modulus determined by Eq. (2), we can find compressional $\left(V_{p}\right)$ and shear $\left(V_{s}\right)$ velocities:

$$
V_{p}=\sqrt{\frac{E(1-v)}{(1+v)(1-2 v) \rho}}, \quad V_{s}=\sqrt{\frac{E}{2(1+v) \rho}},
$$

where $\rho$ is the density of the sample.

The extensional attenuation $Q_{E}^{-1}$ in the sample can be estimated as following. The periodic signals, which are detected by axial strain gauges coupled with the aluminium standard and examination specimen, are averaged and subjected to Fourier transform. The resulting complex Fourier transform amplitudes $A_{a l}$ and $A_{s}$, which are calculated at the frequency of the periodic voltage generated by the oscillator, are used to estimate the attenuation $Q_{E}^{-1}$ :

$$
Q_{E}^{-1} \approx \frac{\operatorname{Im}(A)}{\operatorname{Re}(A)},
$$

where $A=\frac{A_{s}}{A_{a l}} \cdot \frac{\left|A_{a l}\right|}{\left|A_{a l}\right|},,\left|A_{a l}\right|$ and $\left|A_{s}\right|$ are the absolute values of the amplitudes $A_{a l}$ and $A_{s}$, corresponding to the signals obtained from the axial strain gauges coupled with the aluminum standard and specimen correspondingly.

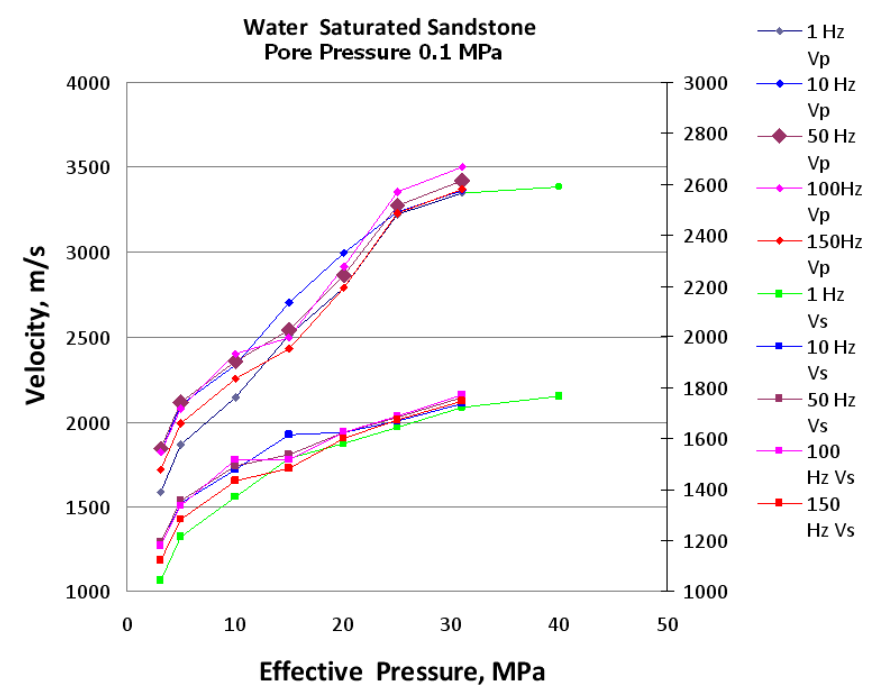

Figure 3: $V_{p}$ and $V_{s}$ velocities obtained for the water saturated sandstone. 


\section{Vienna |'11}

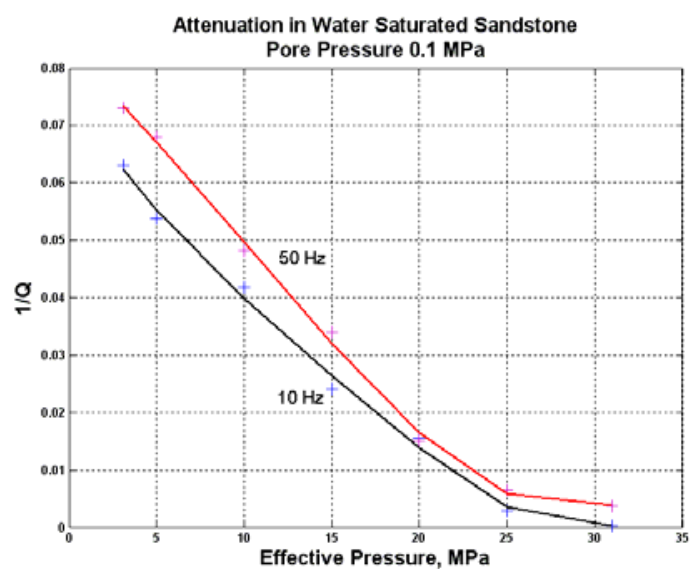

Figure 4: The attenuation 1/Q measured for the water saturated sandstone.

The preliminary results obtained for a sandstone sample at seismic frequencies presented in Figures 3 and 4 . The experiments were carried out at room temperature $\left(\sim 22^{\circ} \mathrm{C}\right)$ and at confining pressures from 3 to $40 \mathrm{Mpa}$. The physical parameters of the water saturated sample are as follows: the density is 2245 $\mathrm{kg} / \mathrm{m}^{3}$, porosity and permeability are $14.8 \%$ and $7.8 \mathrm{mD}$ correspondingly. The diameter of the sample is $38 \mathrm{~mm}$, the length is $70 \mathrm{~mm}$.

\section{Conclusions}

We presented a new version of the low frequency laboratory apparatus operating at seismic frequencies and at either confining or uniaxial pressures from 0 to $70 \mathrm{Mpa}$. The apparatus utilizes a stress-strain technique and measures the complex Young's modulus, extensional attenuation and Poisson's ratio of the rock sample at the range of strain amplitudes between $10^{-6}$ and $10^{-8}$.

\section{References}

Batzle, M.L., Han, D.-H., and Hofmann, R. 2006. Fluid mobility and frequency dependent seismic velocity: Direct measurements. Geophysics, 71(1), N1-N10

Ellis, D.V. and Singer, J.M. Well Logging for Earth Scientist, Second Ed., Springer Science, Dordrecht, The Netherlands, 2008, 692p, p.562.

Paffenholz, J. and Burkhardt, H. 1989. Absorption and modulus measurements in the seismic frequency and strain range on partially saturated sedimentary rocks. J. Geophys. Res., 94(B7), 94939507.

Sheriff, R.E. and Geldart, L.P. Exploration Seismology, Second Edition, Cambridge University Press, Cambridge, 1995, 592p, p.43.

Spencer, J. W. 1981. Stress relaxation at low frequencies in fluid-saturated rocks: Attenuation and modulus dispersion. J. Geophys. Res., 86, 1803-1812.

Weidner, D.J. Elastic Properties of Rocks and Minerals, In: Methods of Experimental Physics, Vol.24 - Part A, Geophysics, Laboratory Measurements, C. G. Sammis and T. L. Henyey (Editors), Academic Press Inc, 1987, 478p, pp.12-13. 